INPLASY

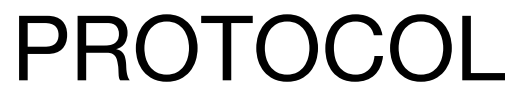

To cite: Liu et al. Efficacy of Vitamin D supplement in Children with Nonalcoholic Fatty Liver Disease: A protocol for systematic review and meta-analysis. Inplasy protocol 202050049. doi:

10.37766/inplasy2020.5.0049

Received: 13 May 2020

Published: 13 May 2020

Corresponding author: Qiu Chen

chenqiu1005@cdutcm.edu.cn

Author Affiliation:

Hospital of Chengdu

University of Traditional Chin

Support: No

Review Stage at time of this submission: Preliminary searches.

Conflicts of interest:

All authors involved in this work have no conflicts of interest.

\section{Efficacy of Vitamin D supplement in Children with Nonalcoholic Fatty Liver Disease: A protocol for systematic review and meta-analysis}

Liu, J1; Wang, TT2; Chen, JM³; Zhang, YL4; Yin, XQ5; Fan, XQ6; Chen, Q; 7 .

Review question / Objective: Population: Patients were eligible for the study if they were children (age $<18$ y) populations of any sex or ethnicity, NAFLD (simple steatosis/ NAFL and NASH); Intervention:Vitamin D supplementation; Compare: Comparisons interventions include placebo control, no therapy, exercise intervention, diet intervention, or active pharmacological treatment. Outcomes:Primary outcomes include effects of Vitamin D supplement on liver fibrosis and liver enzymes;Secondary outcomes include the changings of Serum vitamin D level, BMI, insulin levels, lipid profiles, liver fat content after treatment of Vitamin D supplement. Study: RCTs.

Condition being studied: RCTs of Vitamin D supplementation on Children with Nonalcoholic Fatty Liver Disease.

INPLASY registration number: This protocol was registered with the International Platform of Registered Systematic Review and Meta-Analysis Protocols (INPLASY) on 13 May 2020 and was last updated on 13 May 2020 (registration number INPLASY202050049).

\section{INTRODUCTION}

Review question / Objective: Population: Patients were eligible for the study if they were children (age $<18$ y) populations of any sex or ethnicity, NAFLD (simple steatosis/NAFL and NASH); Intervention:
Vitamin D supplementation; Compare: Comparisons interventions include placebo control, no therapy, exercise intervention, diet intervention, or active pharmacological treatment. Outcomes: Primary outcomes include effects of Vitamin D supplement on liver fibrosis and liver enzymes; Secondary 
outcomes include the changings of Serum vitamin D level, BMI, insulin levels, lipid profiles, liver fat content after treatment of Vitamin D supplement. Study: RCTs.

Condition being studied: RCTs of Vitamin D supplementation on Children with Nonalcoholic Fatty Liver Disease.

\section{METHODS}

Search strategy: Relevant studies were identified in the following electronic databases: PubMed,Google Scholar, Cochrane Library, Web of Science and ClinicalTrials.gov Clinical trial registries were also searched for unpublished trials. All databases were searched from inception to present in English or Chinese .We used combinations of the following key words: (Vitamin D or calcifediol or ergocalciferol or cholecalciferol or 25-hydroxyvitamin $D$ or 1,25-dihydroxy vitamin $D$ or 25hydroxyvitamin D2 or 25(OH)D or 25hydroxycholecalciferol or 1,25(OH) 2D) and (fatty liver or nonalcoholic fatty liver disease or NAFLD or hepatic steatosis or NASH or nonalcoholic steatohepatitis) and (children or Adolescents or Adolescence or Teens or Teen or Teenagers or Teenager or Youth or Youths or Adolescents, Female or Adolescent, Female or Female Adolescent or Female Adolescents or Adolescents, Male or Adolescent, Male or Male Adolescent or Male Adolescents) and (randomised controlled trial or randomized controlled trial or controlled clinical trial or clinical trial or randomized or randomised).

Participant or population: Patients were eligible for the study if they were children(age $<18 y$ ) populations of any sex or ethnicity, NAFLD (simple steatosis/NAFL and NASH).

Intervention: Vitamin D supplementation.

Comparator: Comparisons interventions include placebo control, no therapy, exercise intervention, diet intervention, or active pharmacological treatment.
Study designs to be included: Only RCTS.

Eligibility criteria: RCTs of Vitamin D supplementation on Children with Nonalcoholic Fatty Liver Disease.

Information sources: Relevant studies were identified in the following electronic databases: PubMed, Google Scholar, Cochrane Library, Web of Science and ClinicalTrials.gov Clinical trial registries were also searched for unpublished trials. All databases were searched from inception to present. To identify additional trials, we will hand search the bibliographies of all included studies, as well as any reviews on the topic. We will also search conference proceedings related to this topic.

Main outcome(s): Primary outcomes include effects of Vitamin D supplement on liver fibrosis and liver enzymes.

Additional outcome(s): Secondary outcomes include the changings of Serum vitamin D level, BMI, insulin levels, lipid profiles, liver fat content after treatment of Vitamin D supplement.

Data management: Data for the trials will be extracted independently by two review authors using a standard form. The following information will be included: 1) General information on the publications (title, authors, country, journal name, year of publication, etc), 2) Study methods and characteristics (sample size, design, NAFLD assay methods, randomisation, blinding ,inclusion/exclusion criteria and withdrawals/dropouts). 3) Participants (number of participants, age, gender, racial ancestry ,disease severity, clinical and laboratory parameters). 4) Intervention (dose and type of vitamin D, frequency, duration). 5) Control (no treatment, placebo therapy, exercise intervention, diet intervention, other active treatment). 6) Outcomes (outcome data for the main outcomes and additional outcomes of interest). Extracted data will be compared by two review authors for completeness and accuracy and double-checked by another review author if necessary. The 
original authors will be contacted in case of missing data. Disagreements will be resolved by discussion between the two authors, and further disagreements will be arbitrated by the third author.

Quality assessment / Risk of bias analysis: The risk of bias in the included studies will be assessed independently by two authors and presented in a risk of bias table. The decision will be based on the Cochrane collaboration's tools [ [ [] Higgins JP, Altman DG, Gotzsche PC, et al. The Cochrane Collaboration's tool for assessing risk of bias in randomized trial. BJM 2011;343:d5928. ]] to assess the areas and criteria for the risk of bias. We will evaluate the following domains for risk of bias: sequence generation, allocation sequence concealment, blinding of participants and personnel and outcome assessors, incomplete outcome data, selective outcome reporting and other sources of bias. The assessments will be classified into three levels: low risk, high risk and unclear risk.If there is disagreement about bias, the decision is discussed through two reviewers or with a third reviewer.

Strategy of data synthesis: For continuous data, the mean difference (MD) or standardized mean difference (SMD) will be used to measure treatment effect with $95 \%$ Cl. For dichotomous data, treatment effects are presented as a risk ratio (RR) with $95 \%$ Cl.For each included study, missing data will be gathered by contacting the study author. If the relevant data is not available after contacting the author, the missing relevant data will not be included in the analysis. And a sensitivity analysis will be used to determine whether these missing data affect the results of the metaanalysis. If necessary, we will describe the potential impact of the lack of data on the outcome of the review in the 'Discussion' section.Data synthesis will be performed with Cochrane Review Manager (V.5.3) when a meta-analysis is allowed.If the 12 test is less than $50 \%$, the fixed-effects model will be used for data synthesis. If the 12 test is higher than $50 \%$, the randomeffects model will be conducted for data synthesis. If clinical and methodological heterogeneity is present, a subgroup analysis will be performed to investigate possible sources of statistical heterogeneity. When meta-analysis is not available, we will conduct a systematic narrative synthesis providing information to summarise and explain the characteristics and findings of the included studies.

Subgroup analysis: We plan to carry out following subgroup analysis if sufficient comparable studies are identified. 1)Comparison between dose and type of vitamin $D$, frequency, duration, 2) Comparison between different baseline Serum vitamin D levels. 3)Comparison between NAFL and NASH.

Sensibility analysis: In order to investigate the stability of the results, we will conduct a sensitivity analysis for the outcomes by omitting each of the RCT, or excluding the RCTs with high risk of bias, or excluding the RCTs with missing data.In this way, we will be able to assess the impact of individual studies on the overall results and whether the results are reliable. The conclusions will be compared and discussed according to the analysis results.

Language: We will only include studies published in English and Chinese.

\section{Country(ies) involved: China.}

Keywords: Vitamin D; Nonalcoholic Fatty Liver Disease; protocol; systematic review and meta-analysis.

Dissemination plans: We will publish our results in a peer-reviewed journal.

Contributions of each author:

Author 1 - Jie Liu.

Author 2 - Tingting Wang.

Author 3 - Junmin Chen.

Author 4 - Yanli Zhang.

Author 5 - Xiaoqin Yin.

Author 6 - Xueqin Fan.

Author 7 - Qiu Chen. 